\title{
Ebola vaccine is safe and immunogenic, results show
}

\author{
Anne Gulland
}

London

The first results from a trial of a candidate Ebola virus vaccine show that it is safe and immunogenic, says a paper published online in the New England Journal of Medicine.

Researchers at the University of Oxford said the results showed that the vaccine, the first batch of which has already been shipped to Liberia, was safe for testing in volunteers in west Africa.

Some 60 healthy volunteers were vaccinated from 17 September to 18 November 2014, where three groups of 20 received a low, medium, or high dose. The vaccine was well tolerated at all doses: two of the volunteers reported a moderate fever within 24 hours of receiving the high dose, but the fever subsided within a day.

Participants' levels of antibodies increased over a period of 28 days after vaccination, with no significant difference between the levels at different doses.

The trial is one of several safety trials being conducted in the United Kingdom, the United States, Mali, and Switzerland. The vaccine, a monovalent formulation of a chimpanzee adenovirus type 3 , has been developed by the drug company

GlaxoSmithKline (GSK) and the US National Institutes of Health. The UK arm of the trial is funded by the Wellcome Trust, the Medical Research Council, and the Department for International Development.
Adrian Hill, director of the Jenner Institute at Oxford and the lead investigator, said that the results were encouraging in terms of the vaccine's safety profile. He added, "Larger trials in west Africa will be able to tell us more. We are also currently assessing another option, involving a booster dose, for improving immune response levels."

However, when GSK announced that the first batch of the virus was to be shipped to west Africa some researchers questioned whether trials would be successful because of the declining numbers of patients with Ebola virus disease. ${ }^{2}$ Last week the Liberian health minister, Tolbert Nyenswah, said that just five cases of the disease persisted in the whole country.

World Health Organization data published on 27 January showed a total of 22057 cases of the virus so far in the three worst affected countries of Guinea, Sierra Leone, and Liberia, including 8795 deaths.

Rampling T, Ewer K, Bowyer G, Wright D, Imoukhuede E, Payne R. A monovalen chimpanzee adenovirus Ebola vaccine-preliminary report. N Engl J Med 2015;372. Gulland A. Ebola vaccine to be tested on 30000 volunteers. BMJ 2015;350:h448.

Cite this as: BMJ 2015;350:h524

๑ BMJ Publishing Group Ltd 2015 